\title{
Hybrid meta-heuristics for combinatorial optimization
}

\author{
Tommaso Urli ${ }^{1}$
}

Published online: 23 September 2015

(C) Springer Science+Business Media New York 2015

\begin{abstract}
Combinatorial optimization problems arise, in many forms, in various aspects of everyday life. Nowadays, a lot of services are driven by optimization algorithms, enabling us to make the best use of the available resources while guaranteeing a level of service. Examples of such services are public transportation, goods delivery, university time-tabling, and patient scheduling.

The fields of meta-heuristics, artificial intelligence, and operations research, have been tackling many of these problems for years without much interaction. However, in the last few years, such communities have started looking at each other's advancements, in order to develop optimization techniques that are faster, more robust, and easier to maintain. This effort gave birth to the fertile field of hybrid meta-heuristics. In this thesis, we analyze some of the most common hybrid meta-heuristics approaches, and show how these can be used to solve hard real-world combinatorial optimization problems, and what are the major advantages of using such techniques.
\end{abstract}

This thesis is based on results obtained by working together with many local and international researchers, and published in a number of peer-reviewed papers.

School: University of Udine

\section{Supervisors:}

Luca Di Gaspero

Graduated: Friday, April 4, 2014

Link to full text: http://www.a4cp.org/sites/default/files/tommaso_urli_-_hybrid_metaheuristics_for_combinatorial_optimisation.pdf

Tommaso Urli

Tommaso.Urli@nicta.com.au

1 NICTA, Canberra, Australia 